\title{
Efecto de la asistencia a tutorías en una asignatura del Diplomado en Administración de Empresas de la UNED
}

Elisa Sánchez Godínez

Encargada de la Cátedra de Estadística, Escuela de Ciencias de la Administración, UNED, Costa Rica

elsanchez@uned.ac.cr

Recibido: 07 de junio del 2017

Corregido: 19 de julio del 2017

Aceptado: 04 de agosto del 2017

\begin{abstract}
Resumen
Conocer el efecto de la asistencia a las tutorías presenciales en la aprobación de asignaturas es un insumo importe para los centros educativos a distancia, sobre todo para establecer políticas institucionales en beneficio de los estudiantes. El trabajo pretende mostrar el resultado analizando una muestra de 265 estudiantes matriculados en el primer periodo académico del año 2016, en una asignatura del diplomado en Administración de Empresas de la Universidad Estatal a Distancia de Costa Rica. Los resultados de la aplicación de una regresión logística muestran que las variables: nota de la asignatura requisito, años en la carrera y la asistencia a tutorías presenciales influyen de forma positiva en la aprobación de las asignaturas matriculadas.
\end{abstract}

Palabras clave: Rendimiento académico, rendimiento estudiantil, asistencia pedagógica, apoyo pedagógico, análisis estadístico.

\section{Abstract \\ Effect of assistance to tutorias in a subject of the Diploma in Business Administration of the UNED}

To know the effect of the attendance to the face-to-face tutorials in the approval of subjects, is an important input for distance education centers especially to establish institutional policies for the benefit of students. This paper aims to show this result by analyzing a sample of 265 students enrolled in the first academic period of the year 2016, in a subject of the Diploma in Business Administration of the Universidad Estatal a Distancia, Costa Rica. The results of the application of a logistic regression show that the variables: note of the required subject, years in the career and the attendance of faceto-face tutorials have a positive influence on the approval of the subjects enrolled.

Key words: Academic performance, student performance, pedagogical assistance, pedagogical support, statistic analysis.

\section{INTRODUCCIÓN}

La asistencia de las y los estudiantes a las tutorías presenciales en la educación a distancia es un aspecto de suma importancia para las autoridades universitarias por constituir un apoyo académico y también porque al brindar el servicio se destinan recursos institucionales que se ven reflejados en la contratación 
de los profesores y las profesoras, el pago de sus viáticos, la movilización a los diferentes centros universitarios así como en la disponibilidad de infraestructura y equipo, sobre todo si los centros universitarios se encuentran distribuidos en todo el país como es el caso de la Universidad Estatal a Distancia (UNED), de Costa Rica.

Cuando la mayoría de las y los estudiantes de esta institución se presentan a las tutorías presenciales de forma sostenida, corroboran que el servicio es aprovechado, principalmente por las poblaciones de más bajos recursos económicos y tecnológicos, lo cual justifica el uso de los recursos institucionales. Por el contrario, la no asistencia evidencia un desaprovechamiento del servicio, lo cual podría afectar la aprobación de las asignaturas y causar un ineficiente uso de recursos institucionales.

En la tabla 1 se presenta el comportamiento experimentado en la asistencia a tutorías presenciales en cinco centros universitarios de mayor matrícula en la UNED, en el tercer periodo académico del año 2015. Las cifras muestran un descenso sostenido de la asistencia a las tutorías presenciales en los cinco centros universitarios, evidencian el desgranamiento de los chicos y chicas entre una tutoría y otra. Al tomar como referencia las cifras de asistencia a la primera y cuarta tutoría se observa que el centro universitario de San José experimentó una disminución de 45,3\%, Alajuela 44,1\%, Palmares 46,8\%, Cartago 38,5\% y Heredia $56,7 \%$.

TABLA 1

UNED. Asistencia a tutorías en los cinco centros universitarios de mayor matrícula. Tercer cuatrimestre 2015

\begin{tabular}{lccccc} 
Centro universitario & $\begin{array}{c}\text { Cantidad de } \\
\text { asignaturas }\end{array}$ & $\begin{array}{c}\text { Primera } \\
\text { tutoría }\end{array}$ & $\begin{array}{c}\text { Segunda } \\
\text { tutoría }\end{array}$ & $\begin{array}{c}\text { Tercera } \\
\text { tutoría }\end{array}$ & $\begin{array}{c}\text { Cuarta } \\
\text { tutoría }\end{array}$ \\
San José & 426 & 4129 & 3433 & 2524 & 2257 \\
Alajuela & 89 & 954 & 824 & 526 & 533 \\
Palmares & 84 & 933 & 805 & 659 & 496 \\
Cartago & 90 & 877 & 810 & 614 & 539 \\
Heredia & 67 & 653 & 536 & 405 & 283 \\
\hline
\end{tabular}

Fuente: Centro de Operaciones Académicas.

El propósito del artículo es mostrar los resultados de una indagación que buscó:

a. Determinar si la asistencia a tutorías presenciales está relacionada con el porcentaje de aprobación de la asignatura,

b. si hay una influencia significativa de la tutoría y otras variables en el porcentaje de aprobación y

c. presentar la relación entre las variables mediante un modelo de regresión lineal.

Para el análisis se utilizará la prueba de Chi-cuadrado para establecer relaciones de dependencia entre las variables, así como el análisis multivariable llamado regresión logística para determinar de forma conjunta la relación de las variables que influyen en la aprobación de la asignatura, además de algunas relaciones de probabilidad entre la variable dependiente y las independientes. Se espera que los resultados sirvan de insumo a las autoridades universitarias para generar estrategias de motivación para una mayor asistencia y aprovechamiento de las tutorías. 


\section{REFERENTE TEÓRICO}

En Costa Rica la UNED es una institución de educación a distancia cuya misión es ofrecer educación universitaria principalmente a los sectores más vulnerables de la sociedad y para una inserción laboral equitativa de sus graduados. Con el fin de garantizar el éxito académico de los estudiantes se señalan tres características deseables en ellos: claridad en la definición de sus metas, la determinación de culminar los estudios y ser el protagonista de su proceso de aprendizaje (UNED, 2013).

El modelo pedagógico de la UNED identifica los siguientes componentes esenciales: el estudiante como el constructor de su propio conocimiento, la docencia en donde diversas personas planifican las experiencias de aprendizaje, los contenidos, como la forma de mediatizar al estudiante y el conocimiento. Por otro lado, como componentes transversales se mencionan: la evaluación de los aprendizajes, del currículo y su aplicación y la comunicación en la cual se utilizan medios didácticos y tecnologías (UNED, 2004).

Para el logro de las metas académicas propuestas por los y las estudiantes, la UNED oferta más de 40 carreras de grado cuyas asignaturas se programas en tres cuatrimestres a lo largo del año. En las asignaturas se utilizan materiales escritos y audiovisuales para facilitar los contenidos a los estudiantes, la entrega de la docencia se apoya en plataformas de aprendizaje Moodle.

Como estrategias de comunicación se ofrecen los siguientes espacios: web institucional, correo electrónico, llamada telefónica, videoconferencias, foro de dudas en las plataformas de aprendizaje Moodle, biblioteca, redes sociales y tutorías presenciales, entre otros (UNED, 2016).

Estos espacios de comunicación brindan a los alumnos y alumnas los apoyos académicos apropiados para la construcción de su propio conocimiento, de todos ellos se ha seleccionado la tutoría presencial como objeto de estudio de este trabajo por dos razones principales: en primer lugar porque constituyen un puente directo de comunicación con el estudiante y la estudiante mediante el cual los y las docentes evacuan dudas en tiempo real, en aspectos administrativos y académicos; en segundo lugar porque el proceso académico activa la coordinación de diferentes instancias institucionales, por ejemplo, los centros universitarios los cuales brindan el espacio físico y el equipo, las diferentes escuelas asignando a las y los tutores, la unidad de transportes encargada del traslado de docentes, la Oficina de Tesorería para el pago de viáticos, entre otras instancias.

En la UNED la tutoría presencial es definida como

La interacción académica que existe entre el docente y el estudiante, y los estudiantes entre ellos, quienes, reunidos en un mismo lugar y momento, tienen como objetivo reforzar el autoaprendizaje, mediante acciones académicas, de orientación, técnicas y administrativas, así como la retroalimentación del conocimiento de la disciplina, del abordaje pedagógico y la mediación utilizada (Programa de Apoyo Curricular y Evaluación de los Aprendizajes, 2014, p. 75).

Además, son programadas en casi todas las asignaturas, mayoritariamente se imparten cuatro tutorías presenciales distribuidas a lo largo del periodo académico, en ellas se trabajan los temas que van a ser evaluados en las pruebas escritas o trabajos y se evacuan las dudas que los estudiantes plantean sobre los contenidos.

El personal docente o tutor debe desplazarse hasta el centro universitario en donde se programan las tutorías, la selección de los lugares se realiza tomando como referencia la matrícula de los y las estudiantes. Actualmente, la UNED cuenta con 37 centros universitarios, incluyendo subsedes, distribuidos en todo el territorio nacional. En algunos casos los muchachos y muchachas deben recorrer largas distancias para llegar a los centros educativos, por tanto es importante que los espacios sean aprovechados tanto por los tutores y las tutoras, que al llegar al centro universitario encuentren una buena asistencia 
de sus alumnos y alumnas, como por parte de los y las estudiantes que al llegar al aula la hallen acondicionada y con un tutor o tutora dispuesto o dispuesta a guiar su aprendizaje.

Como se mencionó anteriormente, el proceso llevado a cabo por la universidad para ofrecer las tutorías presenciales cada cuatrimestre, conlleva la activación de diferentes instancias institucionales que deben coordinar desde su programación hasta su ejecución. Por esta razón es de suma importancia que los y las estudiantes aprovechen este recurso asistiendo a los centros universitarios y participando activamente en las tutorías.

\section{¿Qué son las tutorías presenciales y su importancia en el aprendizaje de los estudiantes?}

Algunas instituciones consideran las tutorías como estrategias que ayudan a las y los estudiantes a permanecer en el sistema educativo y a aumentar el rendimiento académico atendiendo lo relacionado con habilidades para el estudio, estabilidad emocional, entre otros aspectos. "En España, la tutoría orienta el aprendizaje autónomo de los alumnos. En el Reino Unido, el modelo de tutoría académica contribuye en los alumnos a resolver sus problemas de aprendizaje" (García, Cuevas, Vales y Cruz, 2012, p.111).

En la educación a distancia las tutorías cobran una importancia relevante por el hecho de que bajo esta modalidad se pretende formar a profesionales autónomos en relación con aspectos propios de la organización del tiempo y del método de aprendizaje, son un espacio para socializar e interactuar y en el cual el personal docente orienta a la población estudiantil en la consecución de sus metas.

Se pueden identificar dos tipos de tutorías: la presencial y a distancia. La tutoría a distancia presenta siete categorías: por correspondencia, telefónica, por radio, por televisión, audio casete, videocasete y por computador (Maya, 1993). Estos son recursos tradicionales que en la actualidad han sido desplazados por la tutoría presencial o virtual.

La tutoría presencial atiende a personas individualmente, a grupos, instituciones, centros regionales o de forma itinerante. Presenta la ventaja de que son flexibles y a la vez dinámicas, agilizan el conocimiento de forma directa, facilitan la retroalimentación, permiten la objetividad de los procesos evaluativos, entre otras ventajas (Maya, 1993).

Maya (1993) también señala diez objetivos que se persiguen con las tutorías presenciales, algunos de ellos son: orientar al estudiante a evacuar sus dudas sobre los materiales, motivarlo para el estudio a distancia, propiciar el trabajo con el análisis de casos, verificar que el estudiante comprenda lo estudiado, socialización de experiencias y comunicación interpersonal entre el docente y el alumno y entre los estudiantes.

\section{¿Cuál es el rol del docente en las tutorías presenciales?}

El papel del docente es muy importante como guía del proceso de aprendizaje de los y las estudiantes. Pagano (2007) define el rol de tutor como "el apoyo temporal que brinda a los alumnos para permitir, en un espacio real o virtual, que éstos ejecuten su nivel justo de potencialidad para su aprendizaje" (p. 3).

También es el nexo o enlace entre la institución y el estudiante, asegurando de esta manera que perciba la presencia de la institución o casa de enseñanza mediante su tutor (Maya, 1993). 
Por la naturaleza de su papel, debe tener empatía con sus alumnos, debe ser proactivo, mantener buena comunicación, ser buen anfitrión y tener experiencia en didáctica para orientar y enseñar al estudiante; además de facilitarles su integración en la modalidad a distancia (Pagano, 2007).

\section{¿Cuál es el rol del estudiante en la tutoría presencial?}

El estudiante como autorregulador de su propio aprendizaje, que conoce su forma de aprender, administra su tiempo, espacio, su ritmo y estilo de aprendizaje, sabe que debe estar preparado para asistir a las tutorías presenciales, debe estudiar los contenidos y formular de antemano sus dudas y consultas. Cuando lo anterior sucede la tutoría es aprovechada al máximo ya que el tutor desarrolla la clase conforme con las preguntas de los y las estudiantes, cumpliendo de esta manera su objetivo.

En educación a distancia el alumno o alumna es el responsable de su propio proceso de aprendizaje, no tiene que asistir a clases de forma sistemática ya que en esta modalidad no existen las clases, sino las tutorías. Los espacios no solo son utilizados para guiar el proceso de aprendizaje y retroalimentar al estudiante, sino también para recibir motivación, socializar con sus tutores y demás compañeros (Maya, 1993).

\section{METODOLOGÍA}

Para la construcción de la base de datos con información de las y los estudiantes matriculados, se utilizaron tres fuentes de información:

1. El Sistema de Administración de Estudiantes (SAE), del cual se extrajeron las siguientes variables: Sexo (SEXO) esta variable toma el valor de "1" si el estudiante es hombre y "2" si es mujer, la variable Año de ingreso a carrera (INGR) en donde se ubica el año de ingreso del estudiante a la carrera y la variable Matrícula con materiales (MAT) la variable guarda una "S" si la o el estudiante tiene los materiales de la asignatura, dado que la está repitiendo, y una " $\mathrm{N}$ " si no los tiene.

2. El Sistema de Notas de la Web (SNW), del cual se obtienen las variables: Nota de asignatura requisito (NREQ): contiene la nota de la asignatura requisito y Nota de la asignatura en estudio (NASIG): almacena la nota de la asignatura que está siendo analizada, ambas en una escala de 0 a 10.

3. Las listas de asistencia a las tutorías presenciales suministradas por las y los tutores que impartieron tutorías en el cuatrimestre de referencia, contiene el nombre de la asignatura, periodo académico, tutoría, el nombre del estudiante y la firma respectiva de asistencia.

Con esta información se elaboró una base de datos compuesta por 265 registros de estudiantes ${ }^{1}$ de un total de 436 matriculados y matriculadas en la asignatura, en el primer cuatrimestre 2016, lo que quiere decir que para este estudio se analiza un $60,8 \%$ del total de registros, dado que se eliminaron aquellos registros incompletos. Esta condición (registro incompleto) es asignada cuando no se cuenta con la información sobre alguna variable extraída de las bases de datos o con la respectiva lista de asistencia a las tutorías. Como ejemplo, se puede indicar que el SNW solo almacena las calificaciones de los estudiantes a partir del año 2015, por tanto no se contó con la información de aquellos que aprobaron esta asignatura antes de ese año, estos casos no fueron considerados en la muestra, en el caso de las listas de tutorías algunas de ellas no fueron aportadas por los tutores por diferentes motivos.

1. Se omite el nombre de la asignatura con el propósito de guardar la confidencialidad de los estudiantes y los tutores. 


\section{Construcción de variables}

Las siguientes cuatro variables fueron construidas a partir de la información extraídas de las bases de datos institucionales y de la lista de asistencia a tutorías.

- Años en carrera (ACAR): para su construcción se realiza una resta entre el año 2016 (año del estudio) y el año de ingreso a la carrera (INGR).

- No repitente (NREP): tiene un valor de "1" cuando el estudiante no repite la asignatura y de "0" en caso contrario. Se construye a partir de la variable matrícula con materiales (MAT), dado que el SAE no identifica de forma directa si el estudiante es repitente en una asignatura.

- Tutorías (TUTO): esta variable almacena un "1" si el o la estudiante asistió al menos a una de las tutorías presenciales y un " 0 " si no fue a ninguna. Se construye a partir de las listas de asistencia a tutorías de cada centro universitario.

- Aprobación (APRO): es una variable de resultado que almacena un "1" si el estudiante obtiene una nota de 7 o superior en la asignatura y un " 0 " en caso contrario. En su construcción se utiliza la variable nota de la asignatura en estudio (NASIG).

Al analizar los datos se utilizó el paquete estadístico MINITAB versión 17 para calcular las medidas de tendencia central y de variabilidad, las relaciones entre variables y el análisis Multivariable correspondiente.

Mientras, en la descripción de los datos se emplearon medidas de tendencia central y de dispersión, tal como la media que indica el valor alrededor del cual se concentran la información, la desviación estándar la cual muestra qué tan alejados o cercanos están de la media y la mediana que identifica el valor central de cada variable conocido como percentil 50.

Por otra parte, se utilizó la prueba de Chi-cuadrado para establecer relaciones en aquellas variables binarias como SEXO, NREP, TUTOR las cuales pueden tener un efecto en la variable respuesta APRO, la prueba se emplea para comprobar si las diferencias en los datos observados es debido al azar o es producto de la influencia de una variable sobre la otra. Por tanto, un valor de " $P$ " bajo, inferior a 0,05 , indica dependencia entre las variables o sea que una variable influye sobre la otra.

Para el análisis Multivariable se usó una regresión logística con el objetivo de estudiar la relación entre una o más variables independientes y una de resultado o dependiente, se utilizó este tipo de regresión dado que la variable dependiente es del tipo binaria y se tienen variables independientes del tipo continuas y binarias. La regresión resulta en un modelo para predecir la variable dependiente. Con los resultados de la regresión también es posible establecer relaciones de probabilidad entre la variable dependiente y las variables independientes.

El estudio centra su análisis en la asignatura del diplomado en Administración de Empresas mencionada, por varias razones: el acceso a información variada de la asignatura como por ejemplo el diseño curricular y las actividades programadas, la información del rendimiento académico de las personas matriculadas y la posibilidad de contar con el apoyo de los docentes para recopilar información no existente sobre la asistencia a tutorías, en cada centro universitario.

La asignatura en estudio se encuentra ubicada en el nivel de diplomado de la carrera de Administración de Empresas de la universidad, pertenece al área disciplinar científica-básica, requiere destrezas en matemáticas y en el uso de Excel, es de naturaleza teórico-práctica. Se utiliza libro de texto y la plataforma de aprendizaje en línea Moodle, donde se accede a materiales audiovisuales que ayudan a reforzar los conocimientos en los contenidos de la asignatura y además se pueden plantear dudas mediante un foro. 
También se imparten tutorías presenciales para evacuar consultas y para que las y los muchachos refuercen sus conocimientos. Dentro del modelo de evaluación cada alumno y alumna debe realizar cuatro tareas, un proyecto y dos exámenes ordinarios. Gran parte de los contenidos aprendidos son aplicados en otras asignaturas del nivel de bachillerato y licenciatura y en el trabajo final de graduación, inclusive.

\section{RESULTADOS}

Las cifras procedentes de las bases de datos institucionales así como la información levantada por cada uno de los y las docentes en las tutorías presenciales, permitieron generar una base de datos consolidada para el análisis. La tabla 2 presenta un resumen de las variables del estudio incluyendo las medidas de tendencia central y dispersión de los datos. En lo que respecta a la variable sexo, la media indica que $63,77 \%$, (169) son mujeres, en relación con la media de años de permanencia en el diplomado establecida en 2,43 años, la mediana refuerza esta cifra al indicar que 50\% (133) tiene 2 años o menos en el diplomado.

Otro dato es que $87,92 \%$ (236) de las y los estudiantes matriculan por primera vez la asignatura en estudio y obtuvieron una nota promedio de 7,9 en la asignatura requisito. Apenas 42,26\% (112) de la población asistieron al menos a una tutoría de las cuatro programadas por cuatrimestre, por último, de un total de 265 estudiantes que matricularon 63\% (167) aprobó la asignatura.

TABLA 2

Análisis descriptivo de las variables

\begin{tabular}{ccccccc} 
Variable & Media & Desviación estándar & Número observaciones & Mínimo & Mediana & Máximo \\
SEXO & 1,6377 & 0,4816 & 265 & 1,0000 & 2,0000 & 2,0000 \\
ACAR & 2,4300 & 2,9970 & 265 & 0,0000 & 2,0000 & 26,0000 \\
NREQ & 7,9585 & 0,9991 & 265 & 0,0000 & 8,0000 & 10,0000 \\
NREP & 0,8792 & 0,3265 & 265 & 0,0000 & 1,0000 & 1,0000 \\
TUTO & 0,4226 & 0,4949 & 265 & 0,0000 & 0,0000 & 1,0000 \\
APRO & 0,6301 & 0,4836 & 265 & 0,0000 & 0,0000 & 1,0000 \\
\hline
\end{tabular}

Fuente: Elaboración propia.

Posterior a la descripción de las variables de estudio, se procedió a determinar la correlación entre ellas para conocer cuáles podían incluirse en el análisis de regresión, utilizando para el análisis la prueba de Chi-cuadrada con el objetivo de determinar la dependencia o independencia de cada una de las variables (NREP, TUTO, SEXO) con la variable respuesta llamada APRO. Los resultados arrojan independencia en las variables SEXO Y APRO debido a que el $P=0,35$ es alto, por tanto la variable sexo no es discriminatoria en la aprobación de la asignatura y no se consideró en la regresión. Por otro lado, la prueba determinó dependencia a un nivel de significancia de 5\% entre NREP Y APRO con una Chi-cuadrada de Pearson de 4,07 y un valor de $P=0,044$, lo que sugiere que la variable NREP podía ser incluida en la regresión.

La prueba de Chi-cuadrada para establecer asociación entre las variables TUTO y APRO fue significativa al 1\%, con un valor de Pearson de 17,88 y un $P$ de 0,000; es decir la asistencia a las tutorías presentó un efecto en la aprobación de la asignatura y por tanto podía ser incluida en la regresión. La tabla 3 muestra la tabla de contingencia entre la asistencia a tutorías y la aprobación de la asignatura. 
Tabla 3

Estudiantes que aprueban o reprueban según asistencia a tutorías. IC 2016

\begin{tabular}{cccccc} 
& \multicolumn{2}{c}{ Valores observados } & \multicolumn{2}{c}{ Valores esperados } & Total \\
\cline { 2 - 5 } Asistencia a tutorías & No aprueba & Aprueba & No aprueba & Aprueba & 153 \\
No & 73 & 80 & 56,58 & 96,42 & 112 \\
Sí & 25 & 87 & 41,42 & 70,58 & 265 \\
Total & 98 & 167 & 98 & 167 & 267 \\
\hline
\end{tabular}

Chi-cuadrada de Pearson $=17,888$ con gl $=1$ y $P=0,000$. Fuente: Elaboración propia.

Al emplear los datos de la tabla de contingencia y realizar algunas divisiones se advirtió que la razón de reprobados entre aprobados cuando se asiste a tutorías es de $0,28=25 / 87$, por ende de cada 100 que aprueban 28 reprueban, el dato mostró la efectividad de la asistencia a tutorías para aprobar la asignatura. Del grupo que no asistió a tutorías, 91 de 100 reprobaron.

El gráfico 1 muestra la comparación de las cifras de asistencia a tutorías con las de rendimiento académicos de las y los alumnos, los datos indican que se presentó un mayor porcentaje de aprobación de la asignatura en los y las estudiantes que asistieron al menos a una tutoría en relación con los que no asistieron, $77,7 \%$ (87 de 112 ) versus $52,3 \%$ (80 de 153) respectivamente.

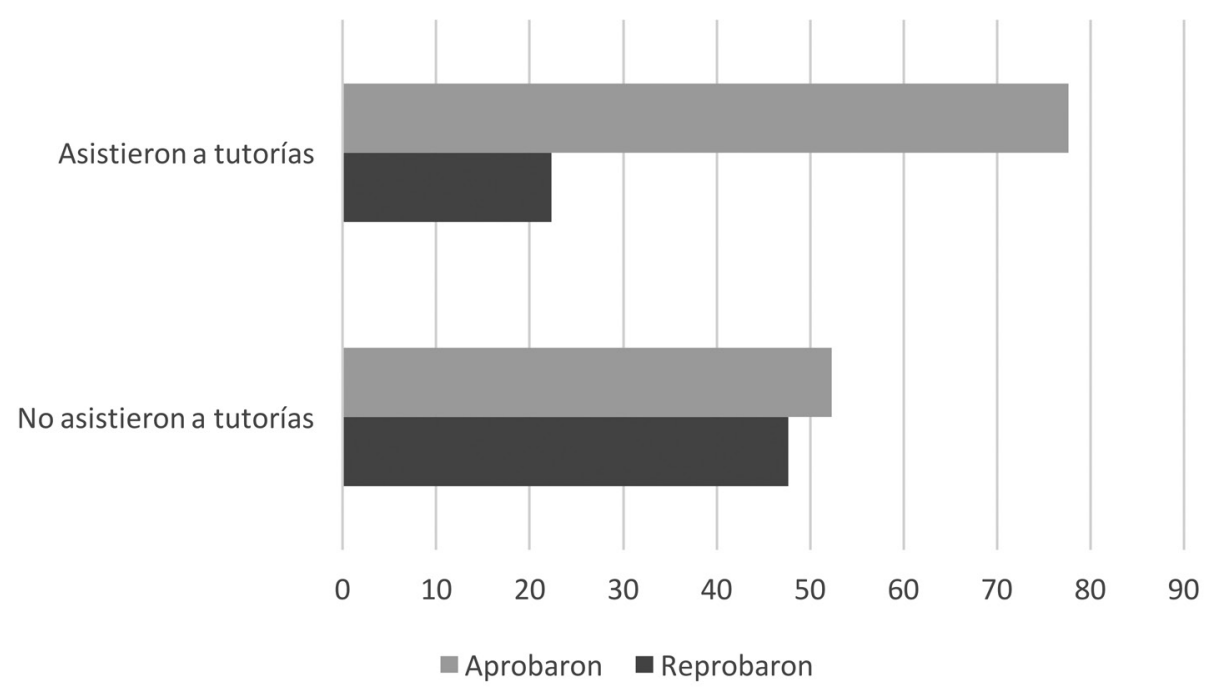

Gráfico 1. Rendimiento académico de los estudiantes que asistieron o no asistieron a tutorías. I cuatrimestre 2016. Fuente: Elaboración propia.

Con la identificación de las variables que mostraron influencia sobre la aprobación de la asignatura en estudio, se procedió a realizar una regresión logística en donde las variables utilizadas en el modelo fueron: NREQ, ACAR, NREP y TUTO. Los resultados de la tabla de desviaciones de la regresión indicaron que las pendientes eran diferentes de cero al nivel de significancia de 1\%, por lo que se concluyó que al menos una variable independiente estaba asociada significativamente con la variable respuesta llamada APRO. 
Los resultados de la regresión arrojaron relaciones significativas de 99\% de nivel de confianza para las variables predictoras: NREQ, ACAR y TUTO, con respecto al evento de referencia que es la aprobación de la asignatura, por otro lado no se encontró una relación significativa entre la variable NREP y la aprobación de la asignatura, por tanto esta variable sale de la regresión.

Los coeficientes positivos del modelo indicaron que en el caso de la asignatura analizada, era más probable que el o la estudiante que hubiese asistido a las tutorías presenciales aprobara la asignatura que el que no asistió, también que entre mayor fuera la nota de la asignatura requisito, más probable sería que el alumno o alumna aprobara la asignatura matriculada. Al estar ubicada en el nivel de diplomado se interpretó que a menor número de años en la carrera mayor fue la probabilidad de aprobar la asignatura.

Las relaciones de probabilidad indicaron que en la asignatura citada, la población estudiantil que asistió a tutorías presenciales tenía una probabilidad 3,43 veces más grande de aprobar la asignatura que la que no fue, suponiendo valores comunes entre las otras variables y que para cada unidad adicional en la nota de la asignatura requisito se contemplaba una probabilidad 3,07 veces más grande de aprobar la asignatura.

Seguidamente, se presentan dos ecuaciones de regresión suministradas por el paquete utilizado para el análisis, una para cada nivel tomado por la variable independiente asistencia o no asistencia a las tutorías.

1. No asistió a tutorías presenciales, tiene una nota " $Q$ " en la asignatura requisito y tiene " $\mathrm{X}$ " años en la carrera: $Y^{\prime}=-8,398+1,122 Q-0,1522 X$

2. Asistió a tutorías presenciales, tiene una nota " $Q$ " en la asignatura requisito y tiene " $\mathrm{X}$ " años en carrera: $Y^{\prime}=-7,166+1,122 Q-0,1522 X$

En términos generales, lo anterior quiere decir que las o los estudiantes que no asistieron a tutorías mostraron una probabilidad menor de aprobar la asignatura. Las pruebas de bondad de ajuste de Pearson y de desviación presentan valores de $p$ superiores al 10\% (0,687 y 0,174 respectivamente), lo que indicó que el modelo se ajusta bien a los datos, con un nivel de significancia del $10 \%$. Por otro lado, la prueba de Hosmer-Lemeshow cuyo $p=0,596$ señaló que existía uniformidad entre las frecuencias observadas y esperadas, en otras palabras, están bastante cerca entre sí.

\section{DISCUSIÓN}

Según muestran los datos de la tabla 1, en general el comportamiento de la asistencia a tutorías impartidas en los centros universitarios de mayor matrícula presenta una tendencia a disminuir entre una tutoría y otra. Un centro universitario como San José que organizó las tutorías de 426 asignaturas en el tercer cuatrimestre 2015, presenta una disminución en la asistencia a tutorías de 696 estudiantes entre la primera tutoría a la segunda, de 909 entre la segunda y la tercera y de 267 entre la tercera y la cuarta tutoría. En el centro universitario de Cartago de 90 asignaturas con tutorías hay una disminución de 67 estudiantes de la primera a la segunda tutoría, de 196 de la segunda a la tercera y de 75 de la tercera a la cuarta.

Este mismo comportamiento se presenta en otros centros universitarios conforme puede observarse en la tabla mencionada, el mayor descenso se ubica entre la segunda tutoría y la tercera. Si bien es cierto, la institución no puede dejar de brindar el servicio de tutorías presenciales ya que beneficia también a estudiantes de bajos recursos, un camino es generar estrategias para aumentar la asistencia.

Tomando como insumo el comportamiento de la asistencia a tutorías en los centros universitarios se realizó una indagación en un grupo de estudiantes matriculados en una asignatura del diplomado en Administración de Empresas de la UNED. El grupo analizado mostró características particulares que lo ubica como una población relativamente joven en la institución, que debe ser orientada con estrategias 
de aprendizaje que les ayuden en la aprobación de asignaturas y en la asimilación de los contenidos. Son mayoritariamente mujeres, situación que va en correspondencia con la misión institucional, el grupo en general presenta un corto tiempo de estancia en el diplomado, en promedio 2,4 años. Una gran mayoría llevó por primera vez la asignatura en estudio y obtuvieron en promedio un 7,9 en la asignatura requisito.

No obstante las condiciones anteriores, se observa un desaprovechamiento de un servicio de apoyo académico brindado por la universidad y que puede ayudarles de manera importante en la aprobación de las asignaturas. La asistencia de estos muchachos y muchachas a tutorías presenciales es relativamente bajo, tan solo $42,26 \%$ de los 265 estudiantes asistieron a evacuar dudas sobre la materia, situación que se reflejó en la aprobación de la asignatura ya que del total de casos analizados tan solo aprobó $63 \%$ (167) de las y los estudiantes.

Por su parte, de la población que asistió a las tutorías presenciales aprobó $77,7 \%$ mientras que de los que no asistieron tan solo aprobó $52 \%$, lo cual sugiere la necesidad de reflexionar acerca de la importancia de las tutorías en la aprobación de asignaturas sobre todo por tratarse de un modelo de educación a distancia en donde se auto gestiona el aprendizaje y las tutorías, como lo señalan algunos autores, favorecen situaciones de aprendizaje.

El modelo de regresión logística aplicado a los datos indicó que la variable nota de asignatura requisito incrementa la probabilidad de aprobar la asignatura. El resultado es esperable dado que en la asignatura requisito se estudian conceptos básicos y técnicas que se emplean en la asignatura en estudio y colaboran en su aprobación. Es importante destacar que las variables: años en la carrera, asistencia a tutorías y nota de asignatura requisito resultaron significativas como variables explicativas de la variable dependiente llamada aprobación.

Del análisis se desprende que en el caso de esta asignatura los muchachos y muchachas que asistieron a tutorías presenciales tienen una probabilidad de aprobación tres veces mayor, en comparación con aquellos que no. El hallazgo debe ser socializado entre la población estudiantil que matricula esta asignatura como una razón de peso para asistir a tutorías y lograr las metas académicas.

\section{CONCLUSIONES}

La investigación analizó una muestra de estudiantes matriculados en una asignatura del Diplomado en Administración de Empresas de la UNED y demostró que la asistencia a tutorías tiene efecto sobre los porcentajes de aprobación. Este servicio universitario cumple una función estratégica importante, guiando al estudiante en su proceso de aprendizaje, aumentando la aprobación de las asignaturas que matriculan y previniendo la deserción.

La universidad y en particular la vicerrectoría académica posee el reto de motivar a las y los estudiantes a que asistan a las tutorías presenciales por dos razones principales: la primera es que contribuye con la aprobación de asignaturas y la segunda es que una mayor asistencia a tutorías representa una justificación importante de la movilización de todas las instancias institucionales involucradas en este proceso, debido a que en oficinas centrales se realiza la contratación de las y los docentes, pago de viáticos y su respectiva movilización a los diferentes lugares, además por parte de los centros universitarios se brinda la disponibilidad de infraestructura y equipo, entre otros apoyos.

Por su parte, tanto los centros universitarios como los y las docentes en general deben motivar a la población estudiantil para que asistan a tutorías haciéndoles ver su importancia en la aprobación de las asignaturas. La motivación podría realizarse mediante anuncios en las plataformas de aprendizaje, en las propias tutorías presenciales o utilizando otros medios de comunicación como Facebook o el 
WhatsApp. Para aquellos estudiantes que no asisten a las tutorías presenciales se les pueden colgar, en la plataforma Moodle, algunas reflexiones sobre la tutoría presencial.

No se debe olvidar que las y los docentes deben ser creativos a la hora de impartir las tutorías para cautivar la atención de los muchachos y muchachas que estudian en un modelo a distancia, para lo cual deben apoyarse en equipo tecnológico y utilizar formatos variados de presentación de los contenidos; se pueden hacer prácticas, formar grupos, plantear preguntas a los alumnos y alumnas y muchas otras dinámicas que generen experiencias de aprendizaje en los estudiantes. También deben ser cordiales y accesibles para despertar la confianza de realizar consultas y de obtener una respuesta satisfactoria. Por otra parte, los centros universitarios velarían porque el equipo y las instalaciones se hallen en buenas condiciones.

Si bien es cierto que los resultados de la investigación evidencian el efecto de las tutorías en la aprobación de la asignatura estudiada, para poder generalizar los resultados a otras asignaturas tanto de la Escuela de Administración como del resto de asignaturas de la UNED, es importante que se realicen otros estudios comparativos e investigaciones sobre los motivos o causas por las cuales los estudiantes no asisten a las tutorías, así como la comparación de la asistencia a tutorías presenciales versus tutorías virtuales.

\section{REFERENCIAS}

Garbanzo, G. (2007). Factores asociados al rendimiento académico en estudiantes universitarios, una reflexión desde la calidad de la educación superior pública. Recuperado de http://www.revistas.ucr.ac. cr/index.php/educacion/article/viewFile/1252/1315

García, R., Cuevas, O., Vales, J. y Cruz, I. (2012). Impacto del Programa de Tutoría en el desempeño académico de los alumnos del Instituto Tecnológico de Sonora. Recuperado de http://redie.uabc.mx/vol14no1/contenido-garciaetal.html

Maya, A. (1993). Orientaciones básicas sobre la educación a distancia y la función tutorial. Recuperado de www.unesco.org/education/pdf/53_21.pdf

Nassif, M., Aparecida, G. y Noronha, V. (2012). Comunicação síncrona no ensino de estatística aplicada à administração: um estudo em uma disciplina semipresencial. Revista de Gestão on ScienceDirect, 2(20), 165-181. doi: 10.5700/rege493

Pagano, C. (2007). Los tutores en la educación a distancia. Un aporte teórico. Recuperado de http://www. uoc.edu/rusc/4/2/dt/esp/pagano.pdf

Programa de Apoyo Curricular y Evaluación de los Aprendizajes. (2014). Glosario de términos curriculares para la Universidad Estatal a Distancia. Recuperado de http://www.uned.ac.cr/conuniversitario/ images/docs_cu/politicas_academicas/ModeloPedagogico_000.pdf

UNED. (abril, 2013). Rectoría: Misión y visión de la UNED. Recuperado de http://www.uned.ac.cr/ rectoria/myv

UNED. (febrero, 2016). Conociendo la UNED: metodología y recursos. Recuperado de http://www. uned.ac.cr/conociendo-la-uned/metodologia-y-recursos

Vergel, O., Martínez, L. y Zafra, T. (2016). Factores asociados al rendimiento académico en adultos. Revista Científica, 2(25), 206-215. doi: 10.14483/udistrital.jour.RC.2016.25.a4 